\title{
ANÁLISE DE FATORES MOTIVACIONAIS EM ESTUDANTES UNIVERSITÁRIOS QUE ADEREM A UM PROGRAMA DE EXERCÍCIO FÍSICO
}

\author{
ANALYSIS OF THE MOTIVATIONAL FACTORS OF \\ UNIVERSITY STUDENTS WHO ADOPT A PROGRAM OF \\ PHYSICAL EXERCISE
}

\author{
Alexsandra Belido Mourão* \\ Daniel Traina Gama** \\ Gustavo Levandoski ${ }^{* * *}$
}

\section{RESUMO:}

O objetivo deste estudo é analisar os fatores motivacionais que levaram os universitários a aderirem ao projeto de extensão "Programa de Treinamento Físico TIME UFGD". O presente estudo tem caráter descritivo/quantitativo e também denominado como pesquisa de campo. Participaram do estudo 101 universitários do sexo masculino, com faixa etária de 18 a 24 anos. A pesquisa foi realizada mediante questionário desenvolvido por Chagas \& Samulski, contendo 26 questões. Os fatores motivacionais mais expressivos para a aderência ao treinamento físico foram: melhorar a qualidade de vida, melhorar ou manter o estado de saúde, aumentar o bem-estar corporal, aumentar a disposição geral. A motivação predominante para a participação dos acadêmicos foi para $95 \%$ melhorar a qualidade de vida.

Palavras-chave: Motivação; Aderência; Universitários; Exercício Físico.

\begin{abstract}
:
This study aim to analyzes the motivational factors that led university students to join the institutional Sports Program TIME UFGD. The methodology adopted to carry this field research is descriptive/quantitative. A total of 101 male college students aged from 18 to 24 participated in the study. The research was carried out using a questionnaire developed by Chagas \& Samulski, it contains 26 questions. The most significant motivational factors founded for adherence to sports training were to: improve the quality of life, improve or maintain the state of health, increase the body's well-being, increase the overall disposition. The predominant motivation for the participation of the academic students was $95.0 \%$ to improve the quality of life.
\end{abstract}

Keywords: Motivation; Adhesion; College students; Physical exercise. 


\section{Introdução}

Atualmente, existe uma ampla evidência de que o exercício físico tem benefícios inquestionáveis para a saúde física, psicológica e social, e a maioria dos indivíduos universitários denotados como a população mais bem informada, consequentemente, já sabe da importância da prática regular de exercícios físicos e seus benefícios. Estudos sugerem que o exercício físico constitui fator determinante para o estado de saúde, independente de sexo, idade e nível socioeconômico. Analisar as motivações de adultos jovens (estudantes universitários e ingressantes ao mercado de trabalho) e sua aderência à prática regular de atividade física é um desafio que pode auxiliar a explanar alguns dos determinantes motivacionais deste comportamento, principalmente nesta fase de transição após a conclusão da educação básica, uma vez que, nesta nova etapa, uma fonte extrínseca de motivação (professor de Educação Física) é cessada (LEGNANI et al., 2011; GUEDES; LEGNANI; LEGNANI, 2012; LEMOS, 2016).

A participação das pessoas em programas de exercício físicos pode estar alicerçada no campo motivacional. A motivação pode ser um fator importante na busca de qualquer meta ou objetivo a ser atingida por um indivíduo. Portanto, a motivação é um determinante para iniciar a prática de exercícios físicos, podendo tanto justificar sua permanência como os casos de abandono, e ser caracterizada como um processo ativo, intencional, o qual depende da interação de fatores pessoais (intrínsecos) e ambientais (extrínsecos) (RYAN; DECI, 2000).

Estudos relatam que boa parte dos universitários são sedentários/inativos e não se envolvem com programas de atividade física (LEMOS, 2016). Há evidências de que a inatividade física já vem desde o ensino básico; o estudo de Moura et al. (2018) evidenciou que $63,1 \%$ dos estudantes de ensino médio são inativos fisicamente após o turno escolar e 57,9\% continuam inativos nos finais de semana. De modo geral, aqueles que não praticam nenhuma atividade física por longos períodos são denominados sedentários, prejudicando não somente a própria, como também a expectativa de vida, já que o estilo de vida sedentário aumenta o risco de doenças não transmissíveis (MENEGUCI et al., 2015). Compreende-se como comportamento sedentário o tempo em que o indivíduo acordado apresenta gasto energético próximo aos níveis de repouso (RAMIRES et al., 2014). Uma pesquisa com estudantes universitários do Rio Grande do Sul apontou que $41 \%$ dos estudantes universitários apresentam um estilo de vida sedentário (LANSINI et al., 2017), ao passo que outro estudo, envolvendo estudantes do Paraná, indicou relação semelhante, 38,8\% (LEVANDOSKI; PILATTI; ZANNIN, 2016). Já na região norte no estado do Acre foi identificado que cerca de $71,3 \%$ dos estudantes universitários são sedentários (FREITAS et al., 2016).

Os estudos sobre a motivação assumem aspectos importantes para a compreensão das diferenças individuais. $\mathrm{O}$ exercício físico e as atividades esportivas organizadas, se praticados regularmente, auxiliam contra doenças e contribuem para uma melhor qualidade de vida, portanto, os fatores motivacionais para as práticas devem ser fortalecidos para que haja aderência ao longo da vida (CERAR; KONDRIČ; SINDIK, 2017).
Tendo como ponto de partida a motivação, na adesão ou evasão, é de conhecimento que estudantes universitários justificam a fraca adesão às atividades físicas/desportivas pela disponibilidade (falta de tempo), os horários das atividades, a distância, enquanto os principais fatores de adesão são: melhorar a condição física, relaxamento e diminuição dos níveis de estresse, diversão, prevenção de doenças e aumento da autoestima (ESTEVES et al., 2017). Já no estudo de Kondrič et al., (2013), verificou-se que os principais fatores motivacionais para que os alunos se envolvam em atividades esportivas são as pressões de saúde, o prazer, a aptidão física, a gestão do estresse e o bem-estar.

Na pesquisa de Legnani et al. (2011), avaliaramse os fatores motivacionais associados à prática de exercício físico de universitários da Universidade Estadual de Londrina, verificando-se, em seus resultados mais expressivos, os motivos: prazer/bemestar, prevenção de doenças e condição física.

Na pesquisa de Medeiros (2012), obtiveram-se, em seus resultados, os fatores motivacionais principais associados à prática, saúde e condicionamento físico em acadêmicos da Universidade Federal de Santa Catarina. Já o estudo de Alexandre (2017) objetivou identificar quais são os fatores motivacionais que levaram os estudantes da Universidade Federal Uberlândia a participarem da equipe de treinamento, e o resultado foi a competência técnica e competição como fatores associados à prática. Por sua vez, o estudo de Sousa (2017) identificou o fator motivacional energia e competência técnica como fonte de motivação principal de alunos da Universidade Federal de Goiás.

Segundo Sindik et al. (2013), o envolvimento do aluno em uma atividade esportiva durante estudos e posteriormente depende tanto da estrutura motivacional do acadêmico quanto das condições e oportunidades disponíveis em um determinado ambiente. $\mathrm{Na}$ Universidade Federal da Grande Dourados (UFGD), localizada na região Centro-Oeste do Brasil, o esporte universitário, a formação de equipes, o treinamento físico, a implementação e avaliação das políticas de esporte e lazer, implementação de projetos de eventos esportivos são administrados pela Divisão de Esportes (DIESP) da Pró-Reitoria de Assuntos Comunitários e Estudantis (PROAE).

A DIESP iniciou, em 2015, a implementação do programa de esporte universitário, e em 2016 foi criado e regulamentado o projeto TIME UFGD. O TIME UFGD tem como objetivo principal a formação e manutenção de equipes competitivas em modalidades esportivas para representação da instituição em eventos esportivos, de extensão universitária, entre outros, oportunizando aos alunos/atletas compromissos como participação em equipes de treinamento permanente, organização e participação de projetos esportivos. O direcionamento do projeto é participativo, formativo e integrativo dos alunos e comunidade acadêmica, sendo que a avaliação de desempenho é realizada por indicadores como frequência dos participantes nas atividades regulares de treinamento, número de eventos atendidos pelas equipes, número de jogos amistosos e oficiais realizados, nível de satisfação dos participantes. O TIME UFGD contempla ainda um programa de bolsas para atletas que beneficiam 100 
alunos por um período anual de 6 meses, sendo divididos nas modalidades basquetebol, futsal, handebol, natação, voleibol, lutas do programa olímpico, tênis de mesa e xadrez. Os atletas do TIME UFGD têm acompanhamento e organização de treinamento das equipes em uma periodicidade de 2 sessões por semana. Para ser beneficiado com a bolsa atleta, os alunos interessados devem se inscrever e passar por um processo seletivo que envolve avaliações de desempenho físico, técnico e tático.

Portanto, o objetivo deste estudo é analisar quais os fatores motivacionais que levaram os universitários a participarem do projeto de extensão "Programa de Treinamento Físico TIME UFGD" da Universidade Federal da Grande Dourados.

\section{Metodologia}

A pesquisa, de caráter descritivo, foi realizada com 101 indivíduos do sexo masculino com idade entre 18 e 24 anos, regularmente matriculados em 22 cursos da Universidade Federal da Grande Dourados que participavam de um Programa de treinamento físico.

O questionário utilizado foi construído por Chagas e Samulski e adaptado por Rocha, Peio e Zaza (2011), contendo 25 questões fechadas e 1 aberta para obter indicativos dos fatores motivacionais dos participantes. $\mathrm{O}$ questionário apresentou uma consistência interna de $\alpha=0,880$ (Alpha de Crombach) obtido em um estudo piloto não publicado. Os participantes foram escolhidos de forma intencional e por acessibilidade. A aplicação do questionário foi com estudantes universitários participantes do treinamento físico da Divisão de Esportes (DIESP) da Universidade Federal da Grande Dourados, no ano de 2018. Utilizou ainda duas variáveis intervenientes para efeito controle aos fatores de risco à saúde: (1) o consumo de tabaco, tendo como respostas fumante e não fumante; e (2) o estado nutricional adotando os critérios da WHO (1998), considerando o IMC $<24,9$ $\mathrm{kg} / \mathrm{m} 2$ como limite entre o peso normal e excesso de peso. As medidas de estatura e massa corporal total foram obtidas através de informação autorreferida cuja validade é apontada por Rech et al. (2010).

$\mathrm{O}$ estudo respeitou as diretrizes referentes às pesquisas envolvendo seres humanos, sendo apreciado pelo Comitê de Ética e Pesquisas da Universidade Federal da Grande Dourados, sob número 57466716.2.0000.5160, de modo que todos participantes assinaram o Termo de Consentimento Livre e Esclarecido. A análise dos dados referente ao questionário foi realizada por meio de estatística descritiva (frequência). Foi realizada uma análise inferencial através do teste não paramétrico de Kruskal Wallis entre as áreas temáticas do curso de graduação e U de Mann Whitney, adotando um valor de $p<0,05$ para comparar as respostas do questionário com variáveis intervenientes como consumo de tabaco, índice de massa corporal, sendo as medidas de massa e estatura autorrelatadas pelos participantes.

\section{Resultados e discussão}

Em relação ao total dos 101 participantes, 71, 17 e 13 são das áreas de Engenharia, Biológicas/Saúde e Humanas, respectivamente, podendo-se observar que os estudantes da área de Ciências Exatas apresentam maior participação entre os estudantes que aderem ao programa de treinamento desportivo.

Em relação ao perfil esportivo dos participantes, $69,3 \%$ praticam outra atividade física, como musculação, crossfit, ciclismo, além do treinamento no programa da universidade e $93,1 \%$ tiveram vivência esportiva competitiva durante o ciclo de vida escolar, sendo handebol, voleibol, futsal e basquetebol as modalidades mais citadas.

A Tabela 1 traz $o$ resultado dos fatores motivacionais avaliados neste estudo, seguido da comparação/associação dos estudantes através de suas áreas de formação acadêmica.

Os resultados apontaram que os principais motivos foram: manter a boa forma, aumentar o bemestar corporal, aumentar o bem-estar psicológico, melhorar ou manter o estado de saúde, aumentar a disposição geral, melhorar a condição física, por prazer pela atividade física, combater o sedentarismo e melhorar a qualidade de vida, que determinam a maior aderência ao programa, contudo, aumentar o status social, emagrecer, por indicações médicas, por incentivo de amigos/família e melhorar a estética foram os fatores de menor relevância para aderência.

Tabela 1 - Fatores motivacionais que levaram os universitários a aderirem um Programa de treinamento físico.

\begin{tabular}{|c|c|c|c|c|c|c|c|c|c|}
\hline \multirow{2}{*}{ Itens do questionário } & \multicolumn{2}{|c|}{$\begin{array}{c}\text { Total } \\
(\mathrm{n}=101)\end{array}$} & \multicolumn{2}{|c|}{$\begin{array}{c}\text { Engenharia } \\
(\mathrm{n}=71)\end{array}$} & \multicolumn{2}{|c|}{$\begin{array}{c}\begin{array}{c}\text { Biológicas } \\
(\mathrm{n}=17)\end{array} \\
\end{array}$} & \multicolumn{2}{|c|}{$\begin{array}{c}\text { Humanas } \\
(\mathrm{n}=13)\end{array}$} & \multirow[t]{2}{*}{ p } \\
\hline & $\begin{array}{l}\text { Não } \\
(\%)\end{array}$ & $\begin{array}{l}\text { Sim } \\
(\%)\end{array}$ & $\begin{array}{l}\text { Não } \\
(\%)\end{array}$ & $\begin{array}{l}\text { Sim } \\
(\%)\end{array}$ & $\begin{array}{l}\text { Não } \\
(\%)\end{array}$ & $\begin{array}{l}\operatorname{Sim} \\
(\%)\end{array}$ & $\begin{array}{l}\text { Não } \\
(\%)\end{array}$ & $\begin{array}{l}\operatorname{Sim} \\
(\%)\end{array}$ & \\
\hline 1. Manter a boa forma & $12,9 \%$ & $87,1 \%$ & 14,1 & 85,9 & 11,8 & 88,2 & 7,7 & 92,3 & n.s. \\
\hline 2. Aumentar a massa muscular & $44,6 \%$ & $55,4 \%$ & 46,5 & 53,5 & 41,2 & 58,8 & 38,5 & 61,5 & n.s. \\
\hline 3. Aumentar o status social & $81,2 \%$ & $18,8 \%$ & 83,1 & 16,9 & 76,5 & 23,5 & 76,9 & 23,1 & n.s. \\
\hline 4. Melhorar a aparência física & $41,6 \%$ & $58,4 \%$ & 35,2 & 64,8 & 64,7 & 35,3 & 46,2 & 53,8 & 0,040 \\
\hline 5. Aumentar o bem-estar corporal & $6,9 \%$ & $93,1 \%$ & 7,0 & 93,0 & 5,9 & 94,1 & 7,7 & 92,3 & n.s. \\
\hline 6. Aumentar o bem-estar psicológico & $10,9 \%$ & $89,1 \%$ & 15,5 & 84,5 & - & 100 & - & 100 & 0,036 \\
\hline 7. Emagrecer & $58,4 \%$ & $41,6 \%$ & 56,3 & 43,7 & 58,8 & 41,2 & 69,2 & 30,8 & n.s. \\
\hline 8. Melhorar ou manter o estado de saúde & $5,9 \%$ & $94,1 \%$ & 4,2 & 95,8 & 11,8 & 88,2 & 7,7 & 92,3 & n.s. \\
\hline 9. Aumentar a disposição geral & $8,9 \%$ & $91,1 \%$ & 9,9 & 90,1 & 11,8 & 88,2 & - & 100 & n.s. \\
\hline 10. Conhecer melhor o próprio corpo & $41,6 \%$ & $58,4 \%$ & 47,9 & 52,1 & 41,2 & 58,8 & 7,7 & 92,3 & 0,026 \\
\hline 11. Melhorar a postura & $38,6 \%$ & $61,4 \%$ & 45,1 & 54,9 & 29,4 & 70,6 & 15,4 & 84,6 & 0,029 \\
\hline 12. Melhorar a condição fisica & $9,9 \%$ & $90,1 \%$ & 8,5 & 91,5 & 17,6 & 82,4 & 7,7 & 92,3 & n.s. \\
\hline 13. Desenvolver mais auto-confiança & $33,7 \%$ & $66,3 \%$ & 43,7 & 56,3 & 17,6 & 82,4 & - & 100 & 0,003 \\
\hline 14. Reduzir o estresse do trabalho & $42,6 \%$ & $57,4 \%$ & 45,1 & 54,9 & 41,2 & 58,8 & 30,8 & 69,2 & n.s. \\
\hline 15. Aumentar o contato social & $31,7 \%$ & $68,3 \%$ & 32,4 & 67,6 & 35,3 & 64,7 & 23,1 & 76,9 & n.s. \\
\hline 16. Por prazer pela atividade fisica & $6,9 \%$ & $93,1 \%$ & 7,0 & 93,0 & 5,9 & 94,1 & 7,7 & 92,3 & n..s. \\
\hline 17. Liberar a tensão do dia-a-dia & $21,8 \%$ & $78,2 \%$ & 21,1 & 78,9 & 23,5 & 76,5 & 23,1 & 76,9 & n.s. \\
\hline 18. Fazer novas amizades & $20,8 \%$ & $79,2 \%$ & 23,9 & 76,1 & 17,6 & 82,4 & 7,7 & 92,3 & n.s. \\
\hline 19. Por indicações médicas & $92,1 \%$ & $7,9 \%$ & 93 & 7,0 & 88,2 & 11,8 & 92,3 & 7,7 & n.s. \\
\hline 20. Por incentivo de amigos/família & $69,3 \%$ & $30,7 \%$ & 70,4 & 29,6 & 64,7 & 35,3 & 69,2 & 30,8 & n.s. \\
\hline 21. Prevenir doenças & $38,6 \%$ & $61,4 \%$ & 42,3 & 57,7 & 35,3 & 64,7 & 23,1 & 76,9 & n.s. \\
\hline 22. Melhorar a estética & $53,5 \%$ & $46,5 \%$ & 54,9 & 45,1 & 52,9 & 47,1 & 46,2 & 53,8 & n.s. \\
\hline 23. Combater o sedentarismo & $25,7 \%$ & $74,3 \%$ & 26,8 & 73,2 & 23,5 & 76,5 & 23,1 & 76,9 & n.s. \\
\hline 24. Sair da rotina & $28,7 \%$ & $71,3 \%$ & 31,0 & 69,0 & 35,3 & 64,7 & 7,7 & 92,3 & n.s. \\
\hline 25. Melhorar a qualidade de vida & $5,0 \%$ & $95,0 \%$ & 4,2 & 95,8 & 5,9 & 94,1 & 7,7 & 92,3 & n.s. \\
\hline
\end{tabular}

Em relação às áreas de ensino, os alunos de Engenharia significativamente valorizam mais a "aparência física" e menos o "bem-estar psíquico" em comparação com as demais áreas. Os estudantes de Ciências Humanas valorizam mais aspectos ligados ao "conhecimento sobre o corpo" e "correção de postura", além do desenvolvimento de "autoconfiança" e "aparência física". Em relação aos estudantes da área de Ciências Biológicas e da Saúde, constatou-se em destaque que a "aparência física" foi inversamente proporcional aos demais estudantes.

$\mathrm{Na}$ Tabela 2, os participantes foram categorizados em relação a dois marcadores de 
comportamentos de risco - consumo de tabaco e estado nutricional -, com o objetivo de se encontrarem aspectos de vulnerabilidade nas representações dos fatores motivacionais. $\mathrm{Na}$ comparação dos participantes (fumantes vs não fumantes), foi verificado que os estudantes fumantes valorizam mais aspectos como emagrecimento, postura e maior contato social em relação aos não fumantes. Da mesma forma, ao se comparar o IMC "eutróficos e sobrepeso", itens emagrecimento, prevenir doenças e combater o sedentarismo, o grupo de estudantes com sobrepeso tende a valorizar mais estes aspectos motivacionais.

Do total de participantes, $27,7 \%$ são fumantes e $37,6 \%$ apresentam indicativo de sobrepeso, mas considerando que os participantes apresentam um nível de atividade física não sedentário, talvez a variável IMC poderia apresentar erro do tipo 1, justamente por não ser possível distinguir qual o tipo de tecido (muscular ou adiposo) o sujeito apresenta em maior evidência. Contudo, $18 \%$ dos participantes apresentam estes dois indicativos simultaneamente (ser fumante e estar em sobrepeso).

Tabela 2 - Comparação dos Fatores motivacionais em relação a resposta "sim" deste item.

\begin{tabular}{|l|c|c|c|}
\hline \multicolumn{1}{|c|}{ Itens do questionário } & $\begin{array}{c}\text { Não Fumantes } \\
(\mathrm{n}=73)\end{array}$ & $\begin{array}{c}\text { Fumantes } \\
(\mathrm{n}=28)\end{array}$ & $\mathbf{p}$ \\
\hline 7. Emagrecer & $65,8 \%$ & $34,2 \%$ & 0,016 \\
\hline 11. Melhorar a postura & $46,5 \%$ & $53,4 \%$ & 0,008 \\
\hline 15. Aumentar o contato social & $38,4 \%$ & $61,6 \%$ & 0,021 \\
\hline & $\begin{array}{c}\text { IMC Normal } \\
(\mathrm{n}=63)\end{array}$ & $\begin{array}{c}\text { IMC Sobrepeso } \\
(\mathrm{n}=38)\end{array}$ & $\mathbf{p}$ \\
\hline 7. Emagrecer & $19,0 \%$ & $78,9 \%$ & $<0,001$ \\
\hline 21. Prevenir doenças & $54 \%$ & $73,7 \%$ & 0,049 \\
\hline 23. Combater o sedentarismo & $66,7 \%$ & $86,8 \%$ & 0,025 \\
\hline
\end{tabular}

Fonte - Autores.

A questão 26 do questionário era aberta, solicitando-se para que os estudantes descrevessem se havia outro motivo - não citado nas alternativas anteriores - que apontasse a aderência ao treinamento físico da DIESP. Assim, foram encontrados motivos como: melhorar o desempenho no esporte, controlar a hiperatividade e melhorar a concentração.

Em relação à categorização dos indivíduos por área, tem-se como resultado os alunos de Ciências Exatas para maior participação e envolvimento nas atividades físicas e a menor participação de universitários de Ciências Humanas e Ciências Biológicas/Saúde, assim como na pesquisa de Sousa e Nahas (2011), em que se estimaram os alunos do sexo masculino de uma universidade pública da Bahia, onde alunos de Ciências Biológicas, Letras e Artes têm maior prevalência em menores níveis de atividade física no lazer se comparados aos alunos de Ciências da Saúde.

No item 3 do questionário, observa-se que o fator "Aumentar o status social" não é motivo de aderência para prática de exercícios físicos/esportivos para $81,2 \%$, de modo que há estudos que corroboram com os mesmos achados (MEDEIROS, 2012; ALEXANDRE, 2017; SOUSA, 2017).

Segundo a Organização Mundial da Saúde, o tabagismo está associado a doenças cardiovasculares, doenças pulmonares, inúmeros cânceres, doenças gastrointestinais, desfechos obstétricos adversos e desenvolvimento de osteoporose. No presente estudo, $27,7 \%$ de acadêmicos são fumantes e cerca de $18 \%$ apresentaram sobrepeso e tabagismo. É importante ressaltar que esses alunos apresentam conhecimento sobre os malefícios do tabaco, mas não abandonam o hábito. Pesquisas demonstram que a maioria dos estudantes fuma porque considera que o hábito de fumar ajuda na redução de stress (GRANVILLEGARCIA et al., 2012; RAMIS et al., 2012; ROSA et al., 2014; ROCCA; DONADONE; OLIVEIRA, 2017). Em vários estudos, notou-se que a atividade física é classificada como fator de proteção contra a prevalência e o consumo de tabaco (MANTILA; GÓMEZ; HIDALGO, 2011; POLONIO; OLIVEIRA; FERNANDES, 2017). Dessa maneira, tem-se a necessidade de incentivar os universitários a cultivarem hábitos saudáveis, tais como a prática de exercícios físicos.

Quando comparados os fatores motivacionais associados ao índice de massa corporal, obtiveram-se os resultados de motivação para eutróficos (combater o sedentarismo), sobrepeso (combater o sedentarismo, emagrecer, prevenir doenças), resultados que se assemelham com achados disponibilizados na literatura. O estudo de Guedes, Legnani e Legnani (2012) procurou associar os motivos para a prática de exercício físico de acordo com o índice de massa corporal dos universitários, revelando-se que nos estudantes eutróficos (condição física, prevenção de doenças, controle de estresse), sobrepeso (condição física, prevenção de doenças, aparência física), obesos (prevenção de doenças, condição física, controle de peso corporal), ao se compararem as duas pesquisas, pode-se observar que a motivação "Prevenção de doenças" se destacou.

Santos, Campos e Campos (2018), em seu estudo sobre motivação para prática de exercícios físicos em estudantes universitários, teve como objetivo identificar os fatores motivadores representados em cinco aspectos: psicológico, interpessoal, saúde, corporal e físico. Notou-se que para o estado nutricional os indivíduos com baixo peso apresentaram menor motivação relacionada a aspectos interpessoais e os eutróficos são mais motivados por fatores psicológicos do que os com sobrepeso. No aspecto saúde, indivíduos com obesidade apresentaram maior motivação do que os com sobrepeso.

Balbinotti et al. (2011) avaliaram alunos do ensino fundamental e médio das escolas do município de Erechim/RS, praticantes regulares de atividades físicas, divididos em três grupos: obesos, com sobrepeso e eutróficos, em que foi encontrado que, para os obesos, o principal fator da prática de exercícios físicos regularmente foi a saúde. Para os alunos de sobrepeso, estética; saúde; prazer; para os eutróficos, saúde, estética, prazer. Na comparação obesos, sobrepeso e eutróficos, a dimensão estética se destacou na diferença entre os grupos de sobrepesos e eutróficos.

Na questão 26, em aberto, obteve-se o motivo de melhorar o desempenho no esporte como fator de motivação, assim como constatou Alexandre (2017) e Sousa (2017), em suas respectivas pesquisas, em que obtiveram como fator motivacional a competência técnica, com estudantes universitários da Universidade Federal de Goiás e Universidade Federal Uberlândia.

Embora haja uma vasta quantidade de estudos sobre os benefícios do exercício físico associados à qualidade de vida e saúde, ainda há uma ampla parcela de indivíduos que não pratica nenhum tipo de exercício 
físico regularmente. Dimensionar a necessidade da prática de exercícios físicos no âmbito universitário ainda se faz necessário, tendo-se como base vários estudos indicando que a população universitária está cada vez mais inativa/sedentária.

Pengpid et al. (2015) apontam, em seu estudo, que $4 \mathrm{em}$ cada 10 estudantes estão fisicamente inativos, assim como no estudo de Lourenço et al. (2016), que apontou que $8 \mathrm{em}$ cada 10 universitários estavam expostos ao comportamento sedentário. Levandoski, Pilatti e Zannin (2016) verificaram que $38,8 \%$ dos estudantes de engenharia são inativos fisicamente em seu período de lazer e 3,7\% apresentaram simultaneamente três fatores de risco (sedentarismo, sobrepeso e tabagismo).

Um dos principais fatores que interferem e que pode mudar essa realidade no comportamento de uma pessoa é a motivação, portanto, se faz necessário uma maior atenção à motivação, à intenção e ao interesse de cada indivíduo, para que haja uma maior aderência e menor abandono nas atividades físicas/desportivas.

\section{Considerações finais}

$\mathrm{O}$ estudo evidenciou que o fator motivacional de maior frequência entre os estudantes universitários que aderiram ao programa institucional de treinamento físico foi a qualidade de vida (95\%). Deste modo, a relevância por descobrir os motivos que levam os estudantes a participar de treinamentos físicos/ esportivos universitário permitirá se entender melhor este comportamento na comunidade estudantil, desvendando seus interesses na tentativa de promover novas ações que estimulem a exercitação física, psicológica e social.

Por fim, espera-se que os resultados aqui encontrados possam subsidiar possíveis estratégias de intervenção, capazes de alertar sobre hábitos negativos que dificultam um estilo de vida saudável e reduzam o comportamento de risco, na tentativa de combater o sedentarismo, sobrepeso e tabagismo entre os estudantes universitários.

\section{Referências}

ALEXANDRE, L. D. C. Esporte universitário: análise dos fatores motivacionais dos atletas da equipe de Handebol da UFU. Monografia (Graduação em Educação Física) Universidade Federal Uberlândia, 2017.

BALBINOTTI, M. A. A.; ZAMBONATO, F.; BARBOSA, M. L. L.; SALDANHA, R. P.; BALBINOTTI, C. A. A. Motivação à prática regular de atividades físicas $\mathrm{e}$ esportivas: um estudo comparativo entre estudantes com sobrepeso, obesos e eutróficos. Motriz: Revista Educação Física (Online), v..17, n.3, p.384-394, 2011.

CERAR, K.; KONDRIČ, M. M.; SINDIK, J. The profiling of university of Ljubljana students according to their motives for exercise participation. Zdr Varst., v.56, n.2, p. 107-114, 2017.
ESTEVES, D.; VIEIRA, S.; BRÁS, R.; O'HARA, K.; PINHEIRO, P. Assessment of physical activity level and healthy behaviour of portuguese university students. Revista Iberoamericana de Psicología del Ejercicio y el deporte, v.12, n.2, p. 261-270, 2017.

FREITAS, T. V.; DALAMARIA, T.; PINTO, W. J.; SOUZA, O. F. Sedentarismo no Lazer em Estudantes da Universidade Federal do Acre. Journal of Amazon Health Science, v.2, n.2, 2016.

GRANVILLE-GARCIA, A. F.; SARMENTO, D. J. D. S.; SANTOS, J. A.; PINTO, T. A.; SOUSA, R. V.; CAVALCANTI, A. L. Smoking among undergraduate students in the area of health. Ciências saúde coletiva, Rio de Janeiro, v. 17, n. 2, p. 389-396, 2012.

GUEDES, D. P.; LEGNANI, R. F. S.; LEGNANI, E. Motivos para a prática de exercício físico em universitários de acordo com o índice de massa corporal. Revista Brasileira Atividade Física e Saúde, Pelotas/RS, v.17, n.4, p.270-274, 2012.

GUEDES, D. P.; LEGNANI, R. F. S.; LEGNANI, E. Motivos para a prática de exercício físico em universitários e fatores associados. Revista Brasileira de Educação Física e Esporte, v. 26, p. 679-689, 2012.

KONDRIČ, M. M.; SINDIK, J.; FURJAN-MANDIĆ. G.; SCHIEFLER, B. Participation motivation and student's physical activity among sport students in three countries. $\mathbf{J}$ Sport Sci Med., v.12, p.10-8, 2013.

LANSINI, L. C.; DIAS, C. P.; OESTREICH, M. G.; ROSA, L. R.; TIGGEMANN, C. L. Nível de sedentarismo entre estudantes universitários do Rio Grande do Sul e os possíveis fatores associados. Revista O Mundo da Saúde, São Paulo, v.41, n.3, p.267-274, 2017.

LEGNANI, R. F. S.; GUEDES, D. P.; LEGNANI, E.; BARBOSA FILHO, V. C.; CAMPOS, W. Fatores motivacionais associados à prática de exercício físico em estudantes universitários. Revista Brasileira de Ciências do Esporte, Florianópolis, v. 33, p. 761-772, 2011.

LEMOS, F. M. F. Motivação e prática do exercício físico em estudantes do ensino superior. Um estudo realizado no Instituto Politécnico do Porto. Tese (Doutorado em Ciências do Desporto) - Faculdade de Desporto da Universidade do Porto, 2016.

LEVANDOSKI, G.; PILATTI, L. A.; ZANNIN, P. H. T. Quality of Life, Physical Activity and Risk Behaviors: A Case Study in Mechanical Engineering Students. Open Journal of Social Sciences, v. 4, p. 19-27, 2016.

LOURENÇO, L. M.; SOUSA, T. F.; FONSECA, S. A.; VIRTUOSO JUNIOR, J. S.; BARBOSA, A. R.

Comportamento sedentário em estudantes Universitários.

Revista Brasileira Atividade Física e Saúde, v.21, n.1, p. 67-77, 2016.

MANTILA, T. S. C.; GÓMEZ, C. A.; HIDALGO, M. M. D. Actividad física, tabaquismo y consumo de alcohol, en un grupo de estudiantes universitarios. Rev. Salud Pública, v.13, n.5, p.748-758, 2011.

MEDEIROS, P. V. Motivos de prática de futsal em universidade pública. Monografia (Graduação em Educação Física) - Universidade Federal de Santa Catarina, Florianópolis, 2012.

MENEGUCI, J.; SANTOS. D. A. T.; SILVA. R. B.; SANTOS, R. G.; SASAKI, J. E.; TRIBESS, S.; DAMIÃO, 
R.; VIRTUOSO JÚNIOR, J. S. Comportamento sedentário: conceito, implicações fisiológicas e os procedimentos de avaliação. Motricidade (C) Edições Desafio Singular, v.11, n.1, p.160-174, 2015.

MOURA, M. F.; FREITAS, J. F. F.; SOUZA, V. M.; LEVANDOSKI, G. Aderência da atividade física e lazer em adolescentes. Rev. Interdisciplin. Promoç. Saúde, Santa Cruz do Sul, v.1, n.1, p. 46-53, 2018.

PENGPID, S.; PELTZER, K.; KASSEAN, H. K.; TSALA, J. P.; SYCHAREUN, V. Physical inactivity and associated factors among university students in 23 low-, middle- and high-income countries. Int J Public Health, v. 60, n.5, p. 539-49, jul. 2015.

POLONIO, I. B.; OLIVEIRA, M.; FERNANDES, L. M. M. Tabagismo entre estudantes de medicina e enfermagem da Universidade Anhembi Morumbi: Prevalência e avaliação da dependência nicotínica e escala de depressão e ansiedade. Arq Med Hosp Fac Cienc Med Santa Casa São Paulo, v.62, v.1, p.12-7, 2017.

RAMIRES, V.; BECKER, L.; SADOVSKY, A.; ZAGO, A.; BIELEMANN, R.; GUERRA, P. Evolução da pesquisa epidemiológica em atividade física e comportamento sedentário no Brasil: atualização de uma revisão sistemática. Rev Bras Atividade Física e Saúde, v.19, n.5, p.529-47, 2014.

RAMIS, T. R.; MIELKE, G. I.; HABEYCHE, E. C.; OLIZ, M. M.; AZEVEDO, M. R.; HALLAL, P. C. Tabagismo e consumo de álcool em estudantes universitários: prevalência e fatores associados. Rev. Bras. Epidemiol., São Paulo, v. 15, n. 2, p. 376-385, 2012.

RECH, C.R.; ARAÚJO, E.D.S.; VANAT, J.R. SelfPerception of Body Image in the Physical Education Course Students. Revista Brasileira de Educação Física e Esporte, v.24, p.285-292, 2010.
ROCCA, J. Z.; DONADONE, J. C.; OLIVEIRA, V. G. A prevalência do tabagismo entre estudantes da Universidade Federal de Mato Grosso - Campus Universitário de Rondonópolis. Rev. Ciênc. Méd. Biol., Salvador, v. 16, n. 1, p. 59-66, 2017.

ROSA, M. I.; CACIATORI, J. F. F.; PANATTO, A. P. R.; SILVA, B. R.; PANDINI, J. C.; FREITAS, L. B. S. D.; REIS, M. E. F. D.; SOUZA, S. L.; SIMÕES, P. W. T. D. Uso de tabaco e fatores associados entre alunos de uma universidade de Criciúma (SC). Cad. saúde colet., Rio de Janeiro, v. 22, n. 1, 2014.

RYAN R. M.; DECI. E. L. Intrinsic and Extrinsic Motivations: Classic Definitions and New Directions. Contemporary Educational Psychology, v. 25, n 1, p. 5467, 2000 .

SANTOS, A. D.; CAMPOS, L. A. D.; CAMPOS, J. A. D. B. Motivação para prática de exercícios físicos em estudantes de Farmácia-Bioquímica. Rev.Ciên.Farm Básica. Apl., Araraquara, v. 39, Supl. 1, 2018.

SINDIK, J.; MANDIĆ, G. F.; SCHIEFLER, B.; E KONDRIČ, $M$. Differences in the structure of motivation for participation in sport activities among sport students in three countries. Kinesiologia Slovenica,1v.9, p.14-31, 2013.

SOUSA, A. R. Fatores motivacionais para a prática do esporte universitário: um estudo exploratório. Monografia (Graduação em Educação Física) - Universidade Federal de Goiás, 2017.

SOUSA, T. F. D.; NAHAS, M. V. Prevalência e fatores associados a menores níveis de prática de atividades físicas no lazer em estudantes de uma universidade pública do Estado da Bahia. Revista Brasileira de Atividade Física e Saúde, v.16, n.4, 2011.

WORLD HEALTH ORGANIZATION. Obesity: Preventing and Managing the Global Epidemic. Geneva, 1998. 